DOI: https://doi.org/10.46296/rc.v5i9.0045

\title{
Comparación de las propiedades antioxidantes del Pleurotus ostreatus antes y después de tratamientos térmicos
}

\section{Comparison of the antioxidant properties of Pleurotus ostreatus before and after thermal treatments}

\author{
García-Vergara Zenia \\ Profesora Instructora del departamento de Ciencia y Tecnología de los Alimentos, \\ Universidad de Camagüey Ignacio Agramonte Loynaz. Camagüey, Cuba. \\ Correo: zenia.garcia@reduc.edu.cu \\ ORCID ID: http://orcid.org/0000-0001-6730-6758 \\ Paneque-Díaz Yurisdan \\ Recién graduado en Adiestramiento. Ingeniero Químico. \\ Universidad de Camagüey Ignacio Agramonte Loynaz. Camagüey, Cuba. \\ Correo: yurisdan.paneque@reduc.edu.cu \\ ORCID ID: http://orcid.org/0000-0003-2161-8814 \\ Macías-González Armando Antonio \\ Estudiante de Licenciatura en Ciencias Alimentarias, \\ Universidad de Camagüey Ignacio Agramonte Loynaz. Camagüey, Cuba. \\ Correo: armando.macias@reduc.edu.cu \\ ORCID ID: https://orcid.org/0000-0002-1646-1413 \\ Rodríguez-Guerrero Daniela de la Caridad \\ Estudiante de Licenciatura en Ciencias Alimentarias, \\ Universidad de Camagüey Ignacio Agramonte Loynaz. Camagüey, Cuba. \\ Correo: daniela.rodriguez@reduc.edu.cu
}

\begin{abstract}
RESUMEN
Pleurotus ostreatus es una especie de hongo comestible distribuido a nivel mundial que reviste importancia, desde el punto de vista comercial por su valor nutricional. Aún son insuficientes los estudios micoquímicos y se conoce poco sobre su relación con las propiedades antioxidantes. La importancia de conocer dichas propiedades radica en que estos compuestos poseen la capacidad de inhibir radicales libres ejerciendo acción en todos los procesos en los que se reduce o detiene el proceso de oxidación. Los antioxidantes como agentes reductores junto con otros agentes reductores de la dieta como: la vitamina C, E y los carotenoides protegen los tejidos del cuerpo. Por lo anterior el objetivo del presente trabajo de investigación es comparar las propiedades antioxidantes del Pleurotus ostreatus antes y después de tratamientos térmicos. Se realizó una recopilación de información a través de una revisión bibliográfica en diferentes bases de datos como Revista SciELO, Redalyc, Scopus y Google Académico para dar respuesta a dicho objetivo. La seta comestible Pleurotus ostratus presenta una excelente capacidad antioxidante, entre las sustancias antioxidantes se pueden mencionar compuestos fenólicos, flavonoides, vitamina, polisacáridos, aminoácidos, esteroles y tocoferoles. Las propiedades antioxidantes del Pleurotus ostreatus se ven afectadas al ser tratados térmicamente.
\end{abstract}

Palabras claves: propiedades antioxidantes, setas comestibles, Pleurotus ostreatus. Información del manuscrito:

Fecha de recepción: 01 de diciembre de 2021.

Fecha de aceptación: 27 de diciembre de 2021.

Fecha de publicación: 06 de enero de 2022. 


\begin{abstract}
Pleurotus ostreatus is a globally distributed edible mushroom species that is commercially important for its nutritional value. Mycochemical studies are still insufficient and little is known about its relationship with antioxidant properties. The importance of knowing these properties lies in the fact that these compounds have the ability to inhibit free radicals, exerting action in all the processes in which the oxidation process is reduced or stopped. Antioxidants as reducing agents together with other reducing agents in the diet such as: vitamin $\mathrm{C}, \mathrm{E}$ and carotenoids protect body tissues. Therefore, the objective of this research work is to compare the antioxidant properties of Pleurotus ostreatus before and after heat treatments. A compilation of information was carried out through a bibliographic review in different databases such as SciELO Magazine, Redalyc, Scopus and Google Academic to respond to this objective. The edible mushroom Pleurotus ostratus has an excellent antioxidant capacity, among the antioxidant substances we can mention phenolic compounds, flavonoids, vitamins, polysaccharides, amino acids, sterols and tocopherols. The antioxidant properties of Pleurotus ostreatus are affected when it is heat treated.
\end{abstract}

Keywords: antioxidants properties, edible mushrooms, Pleurotus ostreatus.

\title{
1. INTRODUCCIÓN
}

En las últimas décadas los hongos comestibles han generado gran atención como producto alimenticio con excelentes propiedades nutritivas y de beneficio a la salud humana. Esto ha sido el resultado de muchos trabajos de investigación enfocados en el estudio de aua propiedades, entre ellas, sus moléculas bioactivas y sus propiedades antioxidantes. Los hongos son importantes en nuestra dieta por su valor nutricional, los cuales incluyen una cantidad alta de proteínas y bajo contenido de lípidos. Recientemente ha sido un alimento atractivo como alimento funcional y como una fuente médica eficiente en terapias contra el cáncer, antiviral, inmunopotenciadora y actividad hipolipidémica. Algunas de estas funciones biológicas fueron atribuidas a su actividad antioxidante (Ramírez, 2009).

La relación que existe entre la concentración de radicales libres y el estado de salud de los seres humanos es un hecho aceptado en la actualidad por la comunidad científica. Los productos derivados de hongos o setas comestibles actualmente presentan los más altos volúmenes de venta en diferentes países. Muchas propiedades medicinales han sido atribuidas a las setas comestibles ya que han demostrado ser reductores del colesterol, poseer efectos antitumorales, antioxidantes, antitrombóticos e inmunomoduladores ("Determinación de las 
potencialidades antioxidantes de Pleurotus $\mathrm{sp}$ in vitro a partir de extractos hidrosolubles", 2020).

Los compuestos antioxidantes pueden interactuar con radicales

libres para evitar que dañen biomoléculas; usando varios mecanismos de acción. La importancia de las propiedades antioxidantes en los alimentos está dada en que estos compuestos poseen la capacidad de inhibir radicales libres (los radicales libres causan enfermedades como asteclerosis, desórdenes en el tracto respiratorio, enfermedades neurodegenerativas y cáncer) ejerciendo acción en todos los procesos en los que se reduce o detiene el proceso de oxidación. Los antioxidantes como agentes reductores junto con otros agentes reductores de la dieta como: la vitamina $\mathrm{C}$, vitamina $\mathrm{E}$ y los carotenoides protegen los tejidos del cuerpo (Salazar, 2017).

Algunos compuestos actúan como agentes antioxidante,entre ellos los compuestos fenólicos y vitaminas, entre otros. Entre los compuestos fenólicos se encuentran: el ácido gálico, ácido cafeico, quercetina y rutina, entre otros. Así también están los flavonoides, con gran capacidad antioxidante y que desempeñan un papel esencial en la protección frente a diversas patologías como la cardiopatía isquémica, la aterosclerosis o el cáncer. Entre los flavonoides encontrados en los hongos se puede citar a la naringenina, la rutina, la morina y de los más conocidos, el resveratrol (Ramírez, 2009).

La gírgola, seta ostra o pleuroto ostra (Pleurotus ostreatus), es una especie de hongo basidiomiceto del orden Agaricales. Se distribuye por gran parte del Holártico, en zonas templadas, aunque se cultiva en muchas partes del mundo. Es comestible, estrechamente emparentado con la seta de cardo (Pleurotus eryngii), que se consume ampliamente por su sabor y la facilidad de su identificación ("Determinación de las potencialidades antioxidantes de Pleurotus sp in vitro a partir de extractos hidrosolubles", 2020).

La mayoría de los compuestos antioxidantes se ven afectados en mayor o menor medida por los procesos industriales a los que se someten los productos alimenticios. Uno de los antioxidantes más afectados es el ácido ascórbico, ya que incluso se utiliza como marcador para evaluar las perdidas nutricionales de 
un proceso nuevo. Los tratamientos térmicos como la esterilización, el enlatado, el escaldado y el secado con aire caliente para deshidratar pueden producir grandes pérdidas (50\%) de este compuesto, dependiendo del tipo de verdura que se esté procesando, normalmente en las frutas las pérdidas son algo menores (Mattila et al., 2002).

El efecto del procesado industrial sobre los diferentes compuestos antioxidantes de las frutas y las verduras no ha sido ampliamente estudiado. Existe deficiencia de información que describa el efecto de los procesos industriales en las propiedades antioxidantes de los hongos o en los compuestos responsables de su actividad (Ramírez, 2009).

Las actividades antioxidantes se miden en cuerpos fructíferos frescos, a pesar de que una parte importante de los hongos cultivados (aproximadamente el 35\% de $A$. bisporus) se somete a procesos industriales como la congelación, el enlatado o la deshidratación para conservar los hongos durante largos procesos de transporte y almacenamiento. Estos tratamientos modifican su composición química, lo que significa que sus propiedades antioxidantes también se vean afectadas (Ramírez, 2009).

El dominio de las transformaciones que sufren las setas comestibles ante los tratamientos térmicos permitirá un mejor aprovechamiento de las mismas, siendo el principal objetivo de esta investigación: comparar las propiedades antioxidantes del Pleurotus ostreatus antes y después de ser sometido a tratamientos térmicos, respondiendo a las investigaciones desarrolladas en el proyecto "Desarrollo de tecnologías para la producción de alimentos a partir de setas comestibles en la cadena agroalimentaria", que gestiona el departamento de Ciencia y Tecnología de los Alimentos de la facultad de Ciencias Aplicadas en la Universidad de Camaguey.

\section{MATERIALES Y MÉTODOS}

Se llevó a cabo una comparación a partir de una revisión bibliográfica de las propiedades antioxidantes del Pleurotus ostreatus antes y después de su exposición a tratamientos térmicos. Se realizó una investigación mediante búsqueda manual en diferentes bases de datos como la Revista SciELO, 
Redalyc, Scopus y Google Académico, utilizando las palabras claves: propiedad antioxidante; seta comestible; Pleurotus ostreatus; tratamientos térmicos. La búsqueda se realizó en los idiomas inglés y español. Se caracterizaron los compuestos antioxidantes presentes en el Pleurotus ostreatus, así como se describió el comportamiento de la actividad antioxidante después de la exposición a tratamientos térmicos.

\section{RESULTADOS Y DISCUSIÓN}

Las propiedades antioxidantes de los hongos que más se consumen (tanto cultivados como silvestres) se han evaluado utilizando la mayoría de los métodos in vitro estandarizados, tales como el TEAC, método del $\beta$-caroteno-ácido linoleico, método de los dienos conjugados, habilidad captadora de radicales hidróxilo o DPPH•, habilidad quelante del ion hierro (poder reductor), inhibición de la oxidación lipídica, etc., y muestran valores intermedios si se comparan con los obtenidos por algunas frutas y verduras. La mayoría de los hongos que más comúnmente se consumen como Agaricus bisporus, Pleurotus ostreatus, Boletus edulis y Lentinus edodes siempre están clasificados dentro de los hongos con propiedades antioxidantes más elevadas en comparación con otras especies menos comunes (Ramírez, 2009).

\subsection{Caracterización de los compuestos antioxidantes presentes en el Pleurotus ostreatus}

\section{Aminoácidos}

Uno de los compuestos más potentes que se ha aislado de varias especies de hongos es la ergotioneína (ERT) (aminoácido natural). Este compuesto está presente en los hongos en una alta concentración si se compara con otras fuentes como el hígado, legumbres, ajo, yema de huevo, trucha, etc., una de las especies que mostraron nivele más altos fueron P. ostreatus (Ramírez, 2009)

\section{Polisacáridos}

El pleurano, un polisacárido que se extrae de Pleurotus ostreatus mejora el estatus antioxidante de las ratas (aumenta las actividades superóxido dismutasa 
(SOD), glutatión peroxidasa (GSH-PX) y glutatión reductasa (GRD) en hígado) y disminuye el efecto que producen las lesiones precancerosas inducidas por dimetilhidrazina en colon de ratas (Bobek y Galbaby, 2017).

Los polisacáridos fúngicos son centro de atención para muchos investigadores por su composición especial en carbohidratos y sus estructuras, ya que éstas aparentemente les confieren importantes propiedades biológicas como agentes antitumorales e inmunomoduladores. Estas fracciones de polisacáridos están normalmente unidas a proteínas formando complejos específicos y también se ha puesto de manifiesto que poseen propiedades antioxidantes, aunque aún no está claro si todas estas actividades biológicas están o no relacionadas (Ramírez, 2009).

\section{Esteroles}

Mattila et al. (2002) y Teichmann et al. (2017) han encontrado presencia del ergosterol en $P$. ostreatus cuya sustancia es el esterol que compone las membranas celulares de los hongos, este compuesto es la principal fuente de esteroles para estos organismos.

\section{Tocoferoles}

Estudios realizados mediante ensayos por Ramírez (2009) indicó que a-tocoferol (familia de los tocoferoles) está presente en todas las especies, pero en concentraciones muy diferentes. C. cibarius, B. edulis y L. edodes muestran las concentraciones más altas, seguidos por L. deliciosus y P. ostreatus. Estos valores coinciden con otros autores como Elmastas et al. (2018) que encuentran valores entre $0,12 \mathrm{mg} / \mathrm{g}$ y $0,13 \mathrm{mg} / \mathrm{g}$ en $\mathrm{L}$. edodes y de $0,24 \mathrm{mg} / \mathrm{g}$ en P. ostreatus.

\section{Compuestos fenólicos}

Los compuestos fenólicos son uno de los productos secundarios más ampliamente distribuidos. La capacidad de estos compuestos para actuar como antioxidante ha sido bien establecida. Los polifenoles son antioxidantes multifuncionales por actuar como agentes reductores, antioxidantes donadores de hidrógeno atrapadores-apagadores de oxígeno. Los hongos por su parte, contienen una variedad de metabolitos secundarios, incluido varios compuestos fenólicos, los cuales han demostrado actuar como excelentes antioxidantes. 
Algunos ejemplos de ello son varios hongos cultivados entre los que se encuentra el Pleurotus ostreatus, los cuales han mostrado significativas actividades antioxidantes y de secuestramiento de radicales libres (Zaragoza, 2011)

Los compuestos responsables de la actividad antioxidante descritos en los hongos aún no se han identificado totalmente. Cheung y Cheung, (2017); Elmastas et al. (2018) correlacionan esta actividad con el contenido de fenoles totales, estas correlaciones se calculan normalmente comparando sólo unas pocas especies de hongos y algunas de ellas están localizadas filogenéticamente muy alejadas las unas de las otras, lo que indica que probablemente sus rutas metabólicas sean muy diferentes, así que deben de contener un gran número de metabolitos bioactivos totalmente distintos.

En la gran variedad de especies de hongos distintas, que presentan una gama amplia de colores, texturas, sabores y formas de cuerpos fructíferos, sus propiedades antioxidantes se deben, en todos ellos, a uno o un par de grupos de compuestos comunes para todas las especies (Zaragoza, 2011)

Según Ramírez (2009), através de la cuantificación de fenoles totales en los cuerpos fructíferos de muchas especies de basidiomicetes diferentes indica que los extractos acuosos contienen una mayor cantidad de compuestos fenólicos que los metanólicos. En especies como B. edulis más del 7\% de los compuestos hidrosolubles son de origen fenólico, siendo también esta especie la que mayor cantidad de fenoles presenta en su extracto metanólico. A. caesarea y L. edodes también muestran un alto contenido fenólico en sus extractos acuosos (más del $5 \%$ ) pero no así en sus extractos metanólicos, donde P. ostreatus y A. bisporus son los siguientes a B. edulis con más del $2 \%$ de fenoles totales extraídos. Unos años más tarde, Elmastas et al. (2018) encontraron niveles más elevados en otras especies como Pleurotus ostreatus y Russula delica $(15,7 \mathrm{mg} / \mathrm{g}-26 \mathrm{mg} / \mathrm{g}$ ps).

El contenido de fenoles totales expresados como ácido gálico en un trabajo presentado por Ferrer et al. (2019) alcanzó valores relativamente menores, entre $36.82 \mu \mathrm{g} \cdot \mathrm{mL}^{\wedge}(-1)\left(0.18 \mathrm{mg} \cdot \mathrm{g}\right.$ de 『biomasa】^(-1) )y $72.23 \mu \mathrm{g} \cdot \mathrm{mL}^{\wedge}(-1) \quad(0.36 \mathrm{mg} \cdot \mathrm{g}$ 
de 【biomasa】^(-1) ) ) para un tiempo de cultivo menor de 10 días. Otro estudio realizado por Cruzano et al. (2020) demostró que en comparación con otras especies $P$. ostreatus alcanzó valores inferiores en cuanto a la actividad de ácido gálico en extracto etanólico $(06.15 \mu \mathrm{g} \pm 01.14 \mu \mathrm{g}$ ácido gálico/mg de extractos).

Como plantea Palacios et al. (2011), en esta especie la presencia de compuestos fenólicos tales como: ácido p-cumárico, ácido felúrico, ácido gálico, ácido gentísico, ácido p-hidroxibenzoico, ácido homogentísico, mirecitina y ácido protocatecuico. Otros autores refieren la presencia de ácido p-hidroxibenzoico, cafeico, ácido siringico y la vanillina (Zaragoza et al., 2011). Beltrán et al. (2021) reporta la presencia de quercetina. También se ha detectado la presencia de un contenido fenólico total de $5.49 \mathrm{~g} / 100 \mathrm{~g}$, así como presencia flavonoides (rutina y crisina) que le dan a Pleurotus ostreatus capacidad antioxidante (Espinosa, 2018).

\section{Vitaminas}

De acuerdo con Cruzano et al. (2020), la presencia en Pleurotus ostreatus de vitaminas como ácido ascórbico (2.395 y $0.6204 \mathrm{~g} / 100 \mathrm{~g}$ DW en fresco y muestras secas, respectivamente), mediante el método de Cromatografía Líquida de Alta Resolución (HPLC)

\section{Actividad enzimática oxidativa del P. ostreatus}

P. ostreatus muestran actividad enzimática oxidativa debida la presencia de peroxidasas y/o polifenoloxidasas en mayor o menor medida. La concentración y actividad de estas enzimas va a influir en la capacidad antioxidante de los extractos acuosos, ya que pueden catalizar reacciones de oxidación de antioxidantes fenólicos (Zaragoza, 2011).

Por lo general el hongo comestible P. ostreatus presenta buena capacidad antioxidante que ha sido demostrado en diferentes estudios realizados sobre la materia prima cruda por diferentes autores. Sin embargo, antes de su ingesta estos hongos se someten a distintos tratamientos térmicos que pueden provocar cambios no sólo en sus propiedades organolépticas sino también probablemente en las propiedades antioxidantes observadas (Ramírez, 2009). 


\section{Actividad antioxidante después de la exposición a tratamientos térmicos}

El uso de agua caliente en tratamientos para los hongos se debe evitar para impedir que suceda el efecto negativo del calor sobre el total de compuestos antioxidantes, ya que, aunque existen indicios de que algunos antioxidantes son termorresistentes, otros son destruidos por altas temperaturas (Palacios et al., 2011).

El corte de los cuerpos fructíferos en pequeños trozos se debe llevar a cabo inmediatamente antes del tratamiento térmico, para evitar que las enzimas oxidativas endógenas presentes en los hongos (polifenoloxidasas y peroxidasas) tengan tiempo suficiente para actuar y degradar los compuestos con actividad antioxidante (Ramírez, 2009).

El calentamiento es un factor importante en los niveles de compuestos fenólicos totales, ya que se observa una disminución en Pleurotus ostreatus. El calentamiento actúa principalmente sobre este compuesto, eliminando su presencia ya que se trata de un compuesto muy termolábil (Salazar, 2017).

Según Vásquez (2009), la cuantificación de polifenoles totales del extracto acuoso del hongo comestible Pleurotus ostreatus de acuerdo a los tipos de secados analizados (secado a $65^{\circ} \mathrm{C}$, escaldado y secado a $65^{\circ} \mathrm{C}$ y secado solar) estadísticamente se encontró diferencia altamente significativa, se determinó que el mayor contenido de polifenoles corresponde al secado a $65^{\circ} \mathrm{C}$ y el tratamiento con menor contenido de polifenoles totales correspondió al escaldado por $1 \mathrm{~min} / 100^{\circ} \mathrm{C}$ a ebullición y secado a $65{ }^{\circ} \mathrm{C}$. Como plantea Teichmann et al. (2017) los tratamientos de microondas y agua en ebullición inactiva la polifenoloxidasa, así mismo se da la pérdida del contenido de antioxidantes reducción del peso de las setas y el encogimiento.

Para determinar que método de deshidratación es más efectivo para minimizar las pérdidas en compuestos antioxidantes o sustancias relacionadas Vásquez (2009), comparó dos procesos de deshidratación utilizando secador de bandejas y la liofilización. Pleurotus ostreatus no mostró diferencias significativas cualquiera que sea el método empleado. Aunque las diferencias entre los dos métodos de deshidratación empleados no han sido importantes, se optó por la 
liofilización ya que es un método más rápido y no implica el calentamiento de la muestra.

Mattila et al. (2002) y Teichmann et al. (2017) verificaron que en todas las temperaturas que estudiaron para el tratamiento de escaldado independientemente de la relación agua/producto empleada y los tiempos de permanencia testeados, las condiciones resultaban suficientes para anular el efecto enzimático de la peroxidasa. Esta es una de las enzimas del Pleurotus ostreatus más resistentes al calor (hasta $71^{\circ} \mathrm{C}$ ) y su inactivación asegura la destrucción de otras más lábiles. Las condiciones mínimas que deben cumplirse son: un tiempo de escaldado de 15 segundos y una relación agua/producto de 3 a 1. El seguimiento de la enzima permitió asegurar que, por encima de los 10 segundos, aun a la temperatura más baja $\left(70^{\circ} \mathrm{C}\right)$, el tratamiento de escaldado es efectivo.

\section{CONCLUSIONES}

Los compuestos fenólicos, tocoferoles, esteroles, polisacáridos, vitaminas y actividad enzimática oxidativa forman parte de las propiedades antioxidantes del Pleurotus ostreatus antes de su exposición a tratamientos térmicos.

Las propiedades antioxidantes del Pleurotus ostreatus se ven afectada a la hora de ser tratados térmicamente, principalmente los compuestos fenólicos y la enzima peroxidasas y/o polifenoloxidasa por calentamiento y tratamientos en agua por ebullición respectivamente.

Tratamientos como la deshidratación por secado a $65^{\circ} \mathrm{C}$, deshidratación por liofilización y escaldado son efectivos y no provoca una significativa disminución de la actividad antioxidante si se lleva a cabo cuidando los parámetros de temperatura y tiempo recomendados.

\section{REFERENCIAS}

Beltrán, Y., Rojas, J., Morales, I., \& Morris, H. (2021). Evaluación in silico del efecto de compuestos fenólicos de Pleurotus ostreatus sobre la enzima 5lipoxigenasa (5- LOX). Revista Cubana de Investigaciones Biomédicas, 40(1).

Recuperado

de 
http://scielo.sld.cu/scielo.php?script=sci_arttext\&pid=S086403002021000200002\&lng=es\&tlng=es

Bobek, P., \& Galbaby, S. (2017). Effect of pleuran (beta-glucan from Pleurotus ostreatus) on the antioxidant status of the organism and on dimethylhydrazine-induced precancerous lesions in rat colon. British Journal of Biomedical Science, 164-168.

Cheung, L. M., \& Cheung, P. C. (2017). Mushroom extracts with antioxidant activity against lipid peroxidation. Life Sciences, 403-409.

Cruzano, A., Reyes, A., Nieto, J., \& Collantes, I. (2020). Estudio de los fitoconstituyentes de Pleurotus ostreatus cultivado en residuos de pulpa de café. Tecnica, 30(2). Recuperado de http://revistas.uni.edu.pe/index.php/tecnia/article/view/806

Determinación de las potencialidades antioxidantes de Pleurotus $\mathrm{sp}$ in vitro a partir de extractos hidrosolubles. (2020). Researchgate. Recuperado 9 de septiembre de 2021, de https://www.researchgate.net/publication/325319392_Determinacion_de _las_potencialidades_antioxidantes_de_Pleurotus_sp_in_vitro_a_partir_ de_extractos_hidrosolubles

Elmastas, M., Isildak, O., Turkekul, I., \& Temur, N. (2018). Determination of antioxidant activity and antioxidant compounds in wild edible mushrooms. Journal of Food Composition and Analysis, 337-345.

Espinosa, E. (2018). Obtención de una harina funcional rica en proteínas de buena calidad biológica y del alto valor nutricio a base de cereales y leguminosas mediante fermentación con Pleurotus ostreatus [Tesis doctoral]. Universidad Autónoma de Nuevo León, Nuevo León, México.

Ferrer, J. C., Mas, S. M., Beltrán, Y., Rodríguez, Y., \& Morris, H. J. (2019). Optimización del medio de cultivo para la producción de biomasa y compuestos fenólicos por Pleurotus ostreatus en fase sumergida utilizando la metodología de superficie de respuesta. Tecnología Química, 39(1), 1-16. Recuperado de http://scielo.sld.cu/scielo.php?script=sci_arttext\&pid=S222461852019000100001

Mattila, P., Lampi, A. M., Ronkainen, R., Toivo, J., \& Piironen, V. (2002). Sterol and vitamin D2 contents in some wild and cultivated mushrooms. Food Chemistry, 293-298.

Palacios, I., Lorenzo, M., Moro, C., \& D’arrigo, M. (2011). Antioxidant properties of phenolic compounds occurring in edible mushrooms. Food Chemistry, 
128(3)

128-674.

Recuperado

de

https://doi.org/10.1016/j.foodchem.2011.03.085

Ramírez, A. (2009). Estudio de las propiedades bioactivas de hongos comestibles para el diseño de productos cárnicos funcionales [Tesis doctoral]. Universidad Autónoma de Madrid, Madrid, España.

Salazar, J. M. (2017). Capacidad antioxidante y caracterización fitoquímica de extractos etanólicos de huitlacoche (Ustilago maydis-Zea mays) crudo y cocido. Revista Mexicana de Ciencias Farmacéuticas, 48(3), 37-47. Recuperado de http://www.redalyc.org/articulo.oa?id=57956616005

Teichmann, A., Dutta, P. C., Staffas, A., \& Jägerstad, M. (2017). Sterol and vitamin D2 concentrations in cultivated and wild grown mushrooms: Effects of UV irradiation. LWT - Food Science and Technology, 40(5), 815-822. Recuperado de https://doi.org/10.1016/j.Iwt.2006.04.003

Vásquez, M. (2009). Caracterización físico-químico proximal y evaluación de la actividad antioxidante del hongo comestible (Pleurotus ostreatus) seco y durante el almacenamiento [Tesis de grado]. Universidad Agraria de La Selva, Tingo María, Perú.

Zaragoza, A. (2011). Análisis de los Compuestos Fenólicos de los Hongos Comestibles Huitlacoche (Ustilago maydis) y Setas (Pleurotus ostreatus) y Evaluación de Algunas de sus Posibles Propiedades Nutracéuticas [Tesis de maestría]. Universidad Autónoma de Aguascalientes, Aguascalientes. 